\title{
Multi-stage Inner Conical Hole Hydraulic Self Driving Rotating Magnetic Field Assisted Electrolytic Machining
}

Lin Tang ( $\square$ tang_lin168@126.com )

Xi'an Technological University

\section{Xingchen $\mathrm{Ge}$}

Xi'an Technological University

Chengjin Shi

Xi'an Technological University

Lifeng Zhang

Xi'an Technological University

Kaige Zhai

Xi'an Technological University

\section{Research Article}

Keywords: multi-stage internal cone hole, electrochemical machining (ECM), fluid power self-drive rotating, technological experiment

Posted Date: November 8th, 2021

DOI: https://doi.org/10.21203/rs.3.rs-1038476/v1

License: (c) (1) This work is licensed under a Creative Commons Attribution 4.0 International License. Read Full License 


\title{
Multi-stage inner conical hole hydraulic self driving rotating magnetic field assisted electrolytic machining
}

\author{
Lin Tang $\cdot$ Xingchen Ge $\cdot$ Chengjin Shi $\cdot$ Lifeng Zhang $\cdot$ Kaige Zhai
}

\begin{abstract}
Aiming at the problem of poor surface quality of multi-stage inner conical hole parts in electrochemical machining, a hydraulic self driving rotating magnetic field assisted electrochemical machining method is proposed, a hydraulic self driving rotating flow field model is established and simulated, and the structure of cathode tail blades is optimized. The simulation results show that when the number of cathode blades is 3 and the thickness of blades is $0.8 \mathrm{~mm}$, When the electrolyte flow rate is not less than $5 \mathrm{~m} / \mathrm{s}$, the impeller at the tail of the cathode mandrel can rotate stably. A hydraulic self driving rotating magnetic field assisted electrochemical machining cathode is designed. When the machining voltage is $10 \mathrm{~V}$, the electrolyte temperature is $30^{\circ} \mathrm{C}$, the electrolyte pressure is $1.6 \mathrm{Mpa}$, the cathode feed speed is $5 \mathrm{~mm} / \mathrm{min}$, and the electrolyte is $5 \% \mathrm{NaCl}+16 \% \mathrm{NaNO}_{3}+4 \% \mathrm{NaClO}_{3}$ composite electrolyte, the comparative experimental study of multi-stage inner conical hole electrochemical machining process with and without rotating magnetic field is carried out, The results show that the surface roughness of the workpiece without magnetic field is $\mathrm{Ra} 0.847 \mu \mathrm{m}$ under the same processing parameters . With the addition of rotating magnetic field, the surface roughness of the workpiece is $\mathrm{Ra} 0.437 \mu \mathrm{m}$. The surface quality was improved by $48.41 \%$.
\end{abstract}

Key Words: multi-stage internal cone hole; electrochemical machining (ECM);fluid power self-drive rotating; technological experiment

\section{Introduction}

The integral components of multi-stage inner conical holes with different diameters connected by conical angles are widely used in aerospace, national defense equipment and other fields because of their lightweight and compact structure. The existing machining has technical bottlenecks such as low machining efficiency, poor machining surface quality and difficult to control taper accuracy, which can not meet the actual production needs. It is urgent to solve the problem of high-precision machining of integral components with multi-stage inner conical holes. Rotating magnetic field assisted electrochemical machining is one of the effective methods suitable for multi-stage inner conical hole machining. The slender pull rod is easy to produce vibration when driving the cathode to move and rotate, and the machining stability is poor.

In order to improve the stability of electrochemical machining process and the advanced nature and practicability of electrochemical machining equipment, many researchers and technicians have carried out many research works, and achieved the gradual improvement of system [1]. Due to the influence of cathode design, machine tool system and other factors, there are still some problems, such as unstable machining process and difficult to ensure the forming accuracy of parts, which is the biggest obstacle to the large-scale popularization and application of ECM technology in parts manufacturing. In order to further improve the ECM efficiency and machining accuracy of difficult to machine materials, scholars at home and abroad have done a lot of research work [2-4]. Tang used the combination of orthogonal

Lin Tang $(\stackrel{\nabla}{-}) \cdot$ Xingchen Ge $\cdot$ Chengjin Shi $\cdot$ Lifeng Zhang $\cdot$ Kaige Zhai

School of Mechatronics Engineering, Xi'an Technological University, 710021 Xi'an, People's Republic of China

E-mail: tang_lin168@126.com 
design and grey correlation theory to obtain the composite electrolyte formula suitable for $00 \mathrm{Cr}_{12} \mathrm{Ni}_{9} \mathrm{Mo}_{4} \mathrm{Cu}_{2}$ material, which improved the material removal rate and surface accuracy of electrochemical machining of the material [5]. Qu proposed the electrochemical machining of $\mathrm{Ti}_{6} \mathrm{Al}_{4} \mathrm{~V}$ material with pulsating electrolyte flow field, obtained a high material removal rate, and the surface roughness of the material after machining reached $0.53 \mu \mathrm{m}$ [6]; Burger et al. Studied the electrochemical machining characteristics of LEK94 nickel based single crystal material, improved the surface accuracy of the material, designed a special electrode, and realized the finishing of the conical hole of LEK94 material [7]. Selvarajan et al. Used copper electrode and pulse electrochemical machining method to process Al7075 material, and studied the effects of electrolyte concentration, pulse duty cycle and machining voltage on the actual machining effect [8]. For the research of magnetic field assisted electrochemical machining, various magnetic circuit structures can be designed according to different applications [9]. Domestic and foreign scholars have carried out a series of research on magnetic field assisted electrochemical machining technology from the aspects of simulation and process [10-12]. Professor Fan designed an inner closed multi-body gradual magnetic circuit for magnetic field assisted electrochemical machining, which improved the uniformity and stability of electrolyte flow field and realized high forming accuracy and surface quality [13]. Ning designed different magnetic field superposition methods and analyzed the influence of magnetic field on electrolyte flow field by using Euler Euler two-phase flow model [14]. Professor Tang designed the magnetic gathering magnetic circuit through the magnetic field simulation method, which effectively improved the accuracy of seven station complex cavity ECM and shortened the cathode design cycle [15-16]. Li established the physical and mathematical models of electrolyte flow characteristics and material removal amount by using COMSOL multi physics software, carried out numerical simulation, and verified the model and simulation by experiments [17-19]. Yan designed a rotating magnetic field assisted high frequency group pulse electrochemical machining device, and studied the effects of different magnetic field strength and machining parameters on workpiece surface roughness [20]. The application of computer-aided design and finite element method in cathode design speeds up the development of design work, which can effectively reduce the cost of electrochemical machining and improve the machining efficiency [21-24]. Professor Chen research team established the single-phase fluid turbulence model of blade electrochemical machining process, and obtained a series of research results such as the variation law of anode profile, inter electrode temperature distribution, electrolyte flow rate distribution and electrolyte pressure distribution in the whole electrochemical machining process [25-28]. Ji carried out the combined EDM electrolytic machining of hole expansion. Through the flow field simulation, it is proved that using the external flushing fluid and the corresponding electrode speed, the pipe spiral electrode can increase the maximum flow rate of the gap by $15.6 \%$ [29]. He took the electrochemical machining of round and square holes of titanium alloy as the research object, and discussed the variation law of each physical quantity with machining time [30-31]. Based on the electric field simulation method, Wang Feng studied the electric field in the machining process of three cathode feeding modes: continuous, vibration and pulse vibration coupling, obtained the distribution law of current density on the side of the machined workpiece and the corresponding anodic dissolution rate, and improved the accuracy of TB6 titanium alloy deep and narrow cell electrochemical machining [32]. Li et al. Proposed a jet electrochemical machining method with 
electrode wire in front, and carried out multi physical field simulation of the machining process by using COMSOL software [33].

In this paper, based on ANASY software, the flow field of electrochemical machining is numerically simulated by using dynamic grid technology, and the rotation characteristics of hydraulic self driving rotating magnetic field composite cathode tail impeller are studied. By changing the number of blades and the thickness of blades, the influence of different blade structures on the uniformity of electrolyte flow field when rotating at the lowest electrolyte flow rate is analyzed. At the same time, the rotation law of cathode tail embedded impeller at different electrolyte flow rates is analyzed. The cathode of electrochemical machining assisted by hydraulic self driving rotating magnetic field is designed and the process test is carried out. Finally, the qualified multi-stage inner conical hole sample is machined.

\section{Field true hydraulic self drive of force driven rotation}

The electrolyte flows in from the cathode inlet hole. At first, it flows in a straight line in the inner cavity of the hollow cathode body, and becomes a spiral flow after flowing through and flushing the impeller. When the electrolyte changes from linear flow to helical motion, it will produce rotating dynamic moment. At the same time, the reverse dynamic moment will be generated for the washed impeller. The reverse dynamic moment of the impeller is inversely equal to the rotating dynamic moment of the electrolyte, which can drive the self rotation of the impeller at the tail of the cathode mandrel.

\subsection{Modeling of flow field of force driven hydraulic self driving rotating field}

The flow of electrolyte in the inner cavity of hollow cathode follows three basic laws: mass conservation law, momentum conservation law and energy conservation law.

The research subject of this paper assumes that the fluid medium is water, the water medium is incompressible and its density is constant. Then the mass conservation equation can be expressed as:

$$
\frac{\partial v_{x}}{\partial x}+\frac{\partial v_{y}}{\partial y}+\frac{\partial v_{z}}{\partial z}=0
$$

Where: $v_{x}, v_{y}$ and $v_{z}$ represent the components of velocity $v$ in $x, y$ and $z$ directions.

The differential form of N-S equation can be obtained by expressing the fluid motion equation with velocity component and pressure:

$$
\left\{\begin{array}{l}
\frac{d v_{x}}{d t}=f_{x}-\frac{1}{\rho} \frac{\partial P}{\partial x}+v V v_{x} \\
\frac{d v_{y}}{d t}=f_{y}-\frac{1}{\rho} \frac{\partial P}{\partial y}+v V v_{y} \\
\frac{d v_{z}}{d t}=f_{z}-\frac{1}{\rho} \frac{\partial P}{\partial z}+v V v_{z}
\end{array}\right.
$$

Where: $f_{x}, f_{y}$ and $f_{z}$ respectively represent the components of volume force per unit mass of fluid in $x, y$ and $z$ directions; $v_{x}, v_{y}$ and $v_{z}$ represent the components of fluid velocity $v$ in $x, y$ and $z$ directions respectively; $v$ represents kinematic viscosity $(\mathrm{m} 2 / \mathrm{s}) ; P$ is the dynamic pressure of viscous fluid $(\mathrm{Pa})$. 
Based on the above assumptions, there will be no obvious thermal energy exchange when the impeller is driven to rotate, so the energy conservation equation is ignored in the simulation study. The flow of electrolyte is relatively stable and belongs to steady flow. Considering the computer performance and calculation running time, the effect of gravity is ignored. Based on the six degree of freedom (6DOF) solver, the dynamic grid technology is used to study the influence of high-speed electrolyte flow on the motion characteristics of the impeller. The numerical calculation method based on the dynamic grid technology is as follows:

\section{1) Control equation}

The incompressible fluid continuity equation and N-S equation of Reynolds time averaged method can be expressed as follows:

$$
\begin{gathered}
\frac{\partial u_{i}}{\partial x_{i}}=0 \\
) \\
\frac{\partial u_{i}}{\partial t}+u_{j} \frac{\partial u_{i}}{\partial x_{j}}=-\frac{1}{\rho} \frac{\partial p}{\partial x_{i}}+\frac{\partial}{\partial x_{j}}\left(v \frac{\partial u_{i}}{\partial x_{j}}-\overline{u_{i}{ }^{\prime} u_{j}{ }^{\prime}}\right)
\end{gathered}
$$

The and $u_{i}, u_{j}, p$ in the above equation are time averaged. Where $\frac{\partial}{\partial x_{j}}\left(-\overline{u_{i}^{\prime} u_{j}^{\prime}}\right)$ is the Reynolds stress term.

\section{2)Turbulence model}

The dimension span of the integral component of the multi-stage inner conical hole is large, the cone angle changes greatly, and the flow field in the ECM gap is easy to change suddenly. Through the calculation of Reynolds number, it can be concluded that the flow state conforms to the turbulence model, while the RNG k- $\varepsilon$ turbulence model has good numerical stability in the simulation calculation, and can deal with the flow state with relatively curved flow lines. Based on the above advantages, RNG $\mathrm{k}-\varepsilon$ turbulence model is used in this simulation study.

\section{3) Geometric conservation}

The moving grid shall meet the geometric conservation law, and the calculation equation of the derivative of the control volume to time is:

$$
\frac{d V}{d t}=\oiint_{\partial \Omega}^{\text {ür }} u_{g} \cdot d \stackrel{\text { ur }}{S}=\sum_{n_{f}} \underset{u_{g, j}}{\mathrm{r}} \cdot \stackrel{\mathrm{ur}}{S}_{j}
$$

Where: $n_{f}$ is the number of control surfaces, and $\stackrel{\boldsymbol{m}}{S}_{j}$ is the area vector of $j$ surface.

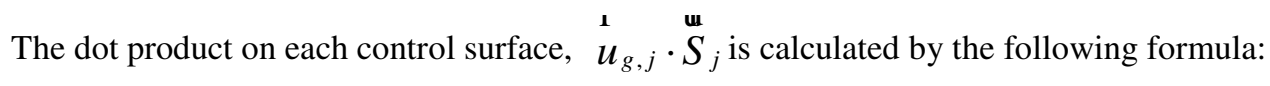

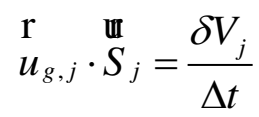

Where: $\delta V_{j}$ is the volume update of control surface $j$ within a time step. 
When establishing the flow field model, due to the complex structure of the hydraulic self driving rotating magnetic field composite electrochemical machining cathode of the multi-stage inner conical hole, in which the mandrel structure in the empty cavity is a step shaft, so both ends of the mandrel are fixed by installing bearings. The cathode machining surface needs to be designed according to the geometry of the multi-stage inner conical hole, including front and rear guide, end cover and other structures, When establishing the model, this simulation can ignore unnecessary geometric elements such as bearings, keep the structure of cathode mandrel and its tail impeller unchanged, and assume that the flow channel of electrolyte is cylindrical. After the model is simplified, it is shown in Figure 1

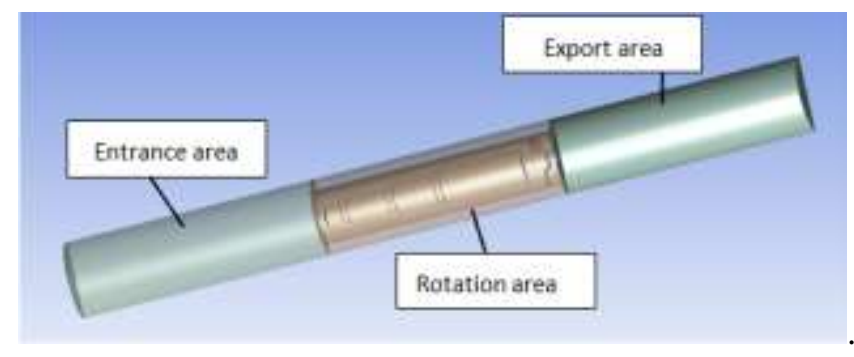

Figure 1 geometric model of flow field

The calculation area of this simulation can be divided into two parts: one is the flow channel area in the whole cathode, and the other is the mandrel area in the cathode cavity. The inlet area of the flow channel is located in the direction of electrolyte inflow in electrochemical machining, and the outlet area is located in the direction of electrolyte outflow. A part of the small area surrounding the mandrel is set as the rotating area. The calculation area of this simulation can be obtained by subtracting the volume of the cathode mandrel from the fluid area of the cathode flow channel.

\subsection{Analysis of simulation results of flow field simulation of force driven hydraulic self driving}

\section{field}

At the beginning of the calculation, the inlet boundary condition is set as the minimum flow rate of electrolyte $5 \mathrm{~m} / \mathrm{s}$. the electrolyte flowing around the inner mandrel of the hollow cathode will go through a stable, rapid change and then tend to be stable. Due to the rotating torque of the impeller rotation, the electrolyte around the cathode inner cavity mandrel changes from the initial linear flow to helical flow, and the flow speed increases sharply. After the electrolyte passes through the cathode mandrel area, the torque is gradually balanced, and the electrolyte is gradually restored to stability.

\section{1) Influence of impeller blade number on the uniformity of flow field}

When the minimum flow rate of electrolyte is $5 \mathrm{~m} / \mathrm{s}$, select $2,3,4$ and 5 blades of the impeller respectively for flow field simulation, set the same calculation time step, and the calculation results of different blade numbers of the impeller at the tail of the mandrel in the cathode cavity on the uniformity of electrolyte flow field are shown in Fig. 2. The calculation results in the figure show that under the same calculation time, when the middle wheel in Figure 2 (a) is 2 blades, the area with the largest electrolyte flow rate appears in the inner wall of the cathode in a point shape, while the area behind the impeller is in the low-speed area, and the flow state of the flow field does not completely change to spiral flow; Fig. 2 (b) when the middle impeller is 3 blades, the flow field behind the impeller rotation area will quickly tend to a stable state, reducing the impact on the cathode mandrel in the rotating state, which is conducive to improving the stability of impeller rotation; In Fig. 2 (c) and Fig. 2 (d), the polarization of electrolyte flow rate is serious, the area of electrolyte low-speed zone behind the impeller is large, and the spiral state of flow field is not obvious. 


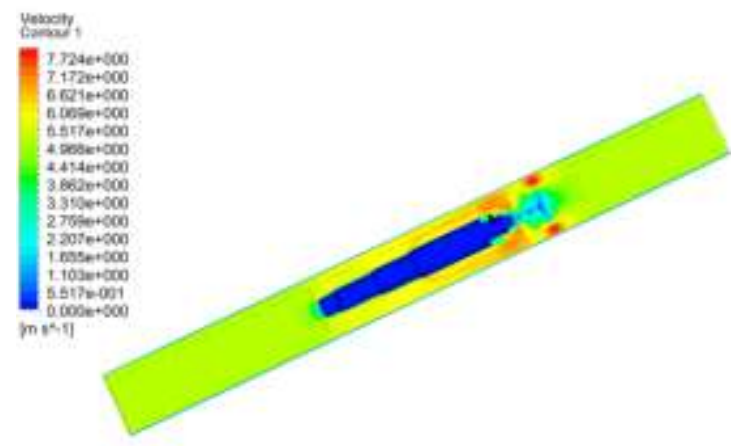

(a) 2 blades

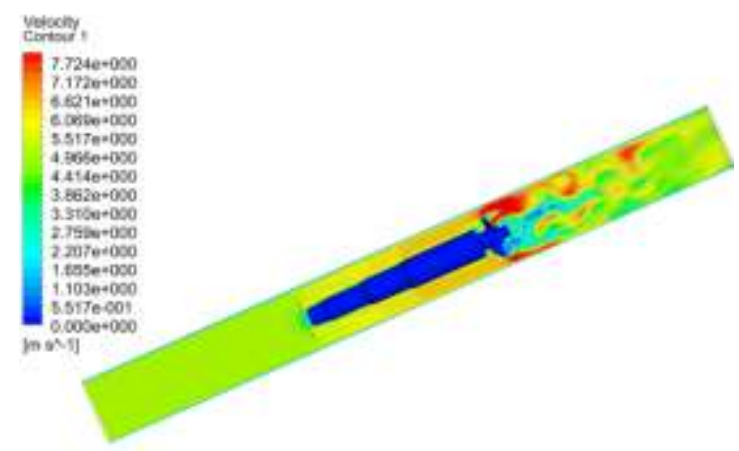

(b) 3 blades

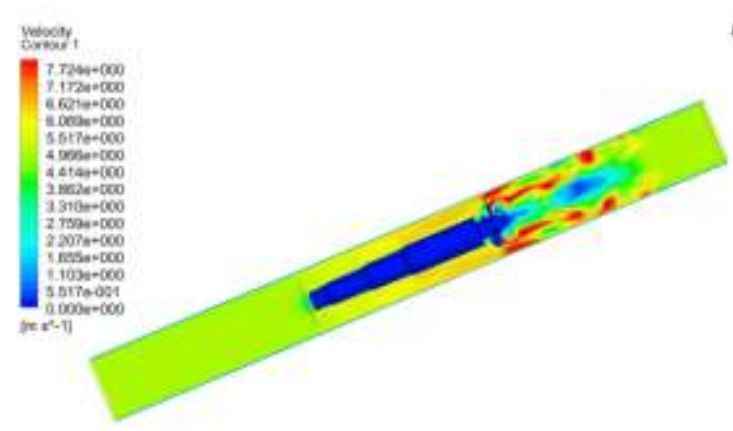

(c) 4blades

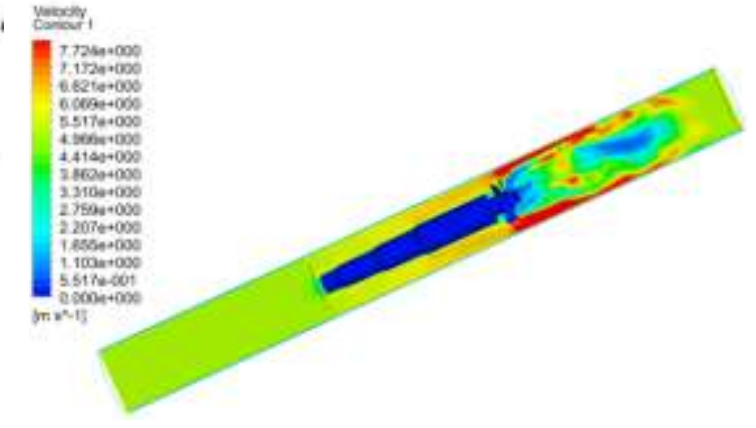

(d) 5 blades

Fig. 2 flow field velocity nephogram of different blade numbers

When the electrolyte flows through the area near the impeller at the tail of the mandrel in the inner cavity of the cathode, the flow of the electrolyte is hindered by the blade body, and the clearance space area between the outer diameter of the impeller and the inner wall of the hollow cathode body is relatively narrow. The pressure of the electrolyte around the impeller suddenly increases, and the flow rate increases. Due to the generation of rotating torque, the flow of the electrolyte presents a spiral flow state, Its reverse torque drives the impeller to rotate. The results of flow field velocity streamline with different number of blades are shown in Figure 3.
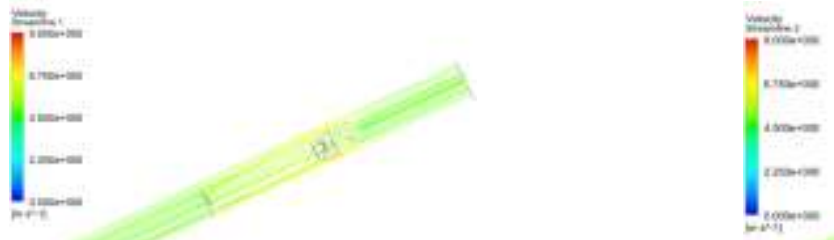

(a) 2blades

(b) 3blades
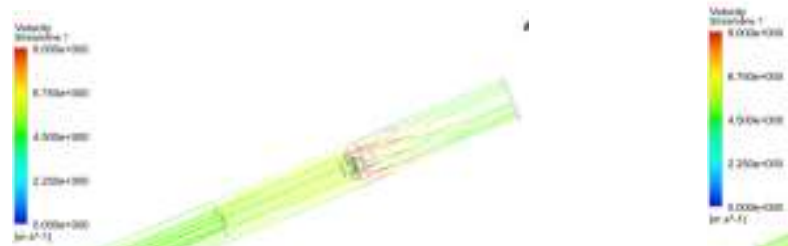

(c) 4blades

(d) 5blades 
Fig. 3 flow field streamline diagram of different blade numbers

The flow of each streamline can be reflected from the figure. In the same calculation time, the calculation results of 2 blades show the initial state of impeller rotation, the flow field streamline has just begun to show the state of spiral flow, and the calculation results of 3 blades have shown that the electrolyte streamline has a stable trend after spiral flow, indicating that when the impeller is of 3 blade structure, the impeller has higher stability than others. Under the same electrolyte flow rate, in the streamline diagrams of 4-blade structure and 5-blade structure, the flow rate of electrolyte in the narrow area between the outer diameter of impeller and the inner wall of cathode presents an extreme value. When the electrolyte flow rate in this area is too large, the inner wall of cathode is under great stress, which is easy to lead to the deformation of cathode structure and affect the forming accuracy of integral components such as multi-stage inner conical holes.

\section{2)Effect of blade thickness on field uniformity}

The electrolyte flow rate is still set as $5 \mathrm{~m} / \mathrm{s}$ and the impeller is 3 blades. The influence of different blade thickness on the uniformity of hydraulic self driving flow field is simulated and analyzed. The blade thickness is designed as $0.5 \mathrm{~mm}, 0.8 \mathrm{~mm}, 1.1 \mathrm{~mm}$ and $1.4 \mathrm{~mm}$ respectively. The distribution of electrolyte flow field is shown in Fig. 4.

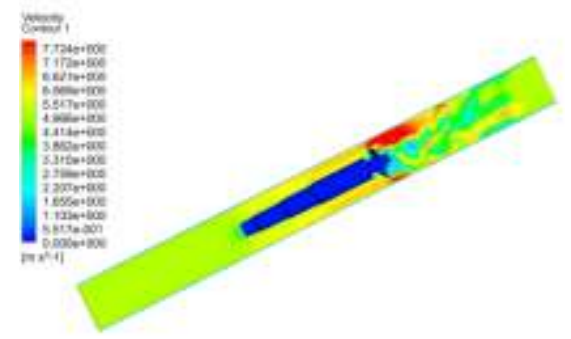

(a) $0.5 \mathrm{~mm}$

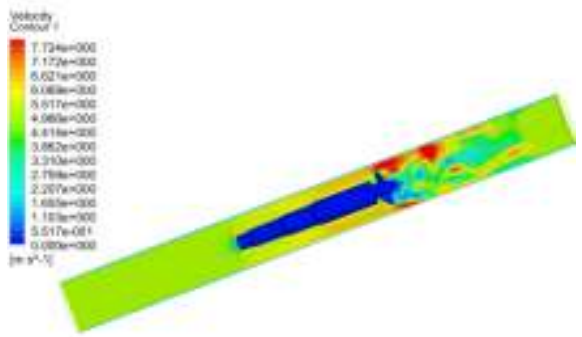

(c) $1.1 \mathrm{~mm}$

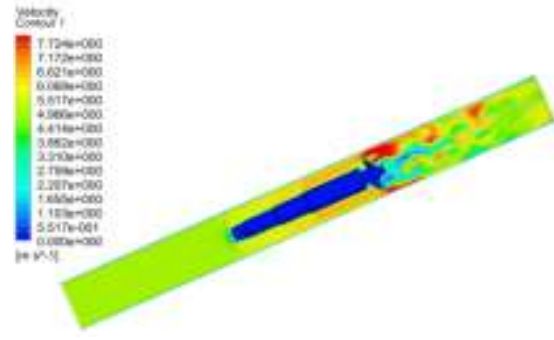

(b) $0.8 \mathrm{~mm}$

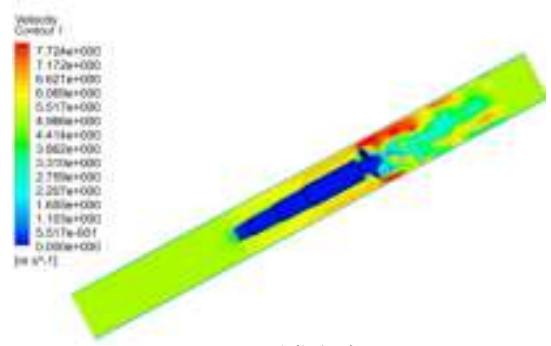

(d) $1.4 \mathrm{~mm}$

Fig. 4 velocity nephogram of different blade thickness

In Fig. 4 (a), the blade thickness is $0.5 \mathrm{~mm}$, the velocity of electrolyte near the impeller close to the inner cavity wall of the cathode is asymmetric, and the spiral flow state is uneven; In Fig. 4 (c), the blade thickness is $1.1 \mathrm{~mm}$, and the flow velocity uniformity of electrolyte flow field behind the impeller is poor. Too high flow velocity will impact the inner cavity wall of cathode and affect the stiffness of cathode body; In Fig. 4 (d), the blade thickness is $1.4 \mathrm{~mm}$, the low-speed area of the electrolyte flow field is large, the high-speed electrolyte is mainly distributed on the side wall of the cathode inner cavity, the spiral flow state is unclear and the velocity difference is too large; Fig. 4 (b) when the blade thickness is $0.8 \mathrm{~mm}$, the spiral flow state of the flow field behind the impeller is more uniform than others, and the polarization of velocity is weak.

The streamline distribution of the flow field is shown in Figure 5. It can be seen from streamline figure 5 (b) that the flow field tends to be gradually stable after the rotation of the impeller. The spiral flow state of the streamline of the other three impeller thicknesses in Figure 5 is relatively disordered. 
The flow velocity close to the side wall of the cathode cavity is too large and the circumferential distribution is uneven, which will cause uneven stress on the side wall of the cathode cavity and deformation, Thus, the cathode working face is affected, and even the forming accuracy of multi-stage inner conical hole is reduced
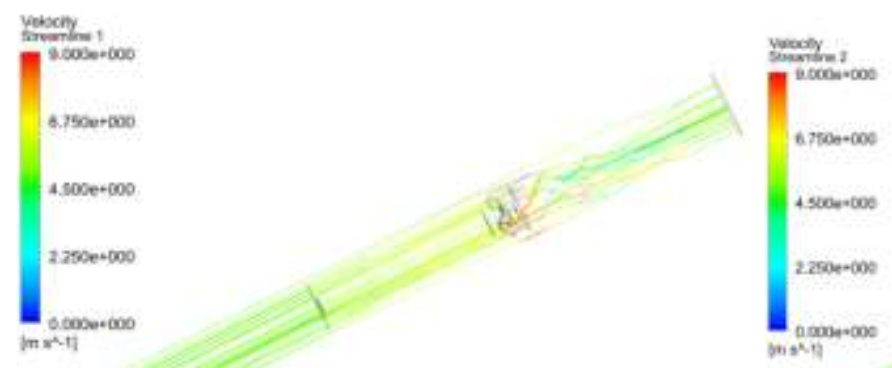

(a) $0.5 \mathrm{~mm}$

(b) $0.8 \mathrm{~mm}$
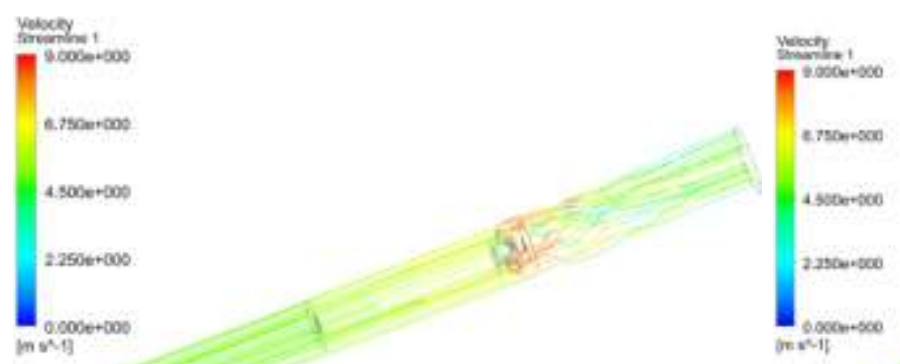

(c) $1.1 \mathrm{~mm}$

(d) $1.4 \mathrm{~mm}$

Fig. 5 flow field streamline of different blade thickness

\section{3) Effect of electrolyte inlet velocity on impeller speed}

In order to study the influence of electrolyte inlet velocity on the rotating state of impeller, the simulation study was carried out under the condition that the impeller is 3 blades and the blade thickness is $0.8 \mathrm{~mm}$. When the inlet velocity of electrolyte is $5 \mathrm{~m} / \mathrm{s}, 6 \mathrm{~m} / \mathrm{s}, 7 \mathrm{~m} / \mathrm{s}$ and $8 \mathrm{~m} / \mathrm{s}$ respectively, observe the distribution of electrolyte flow field around the inner cavity mandrel of hollow cathode. The simulation results are shown in Fig. 6. It can be seen from the figure that when the electrolyte inlet speed is $5 \mathrm{~m} / \mathrm{s}$, the maximum flow rate after flowing through the impeller is $7.724 \mathrm{~m} / \mathrm{s}$, while when the electrolyte inlet speed increases to $8 \mathrm{~m} / \mathrm{s}$, the maximum flow rate after flowing through the impeller reaches $11.59 \mathrm{~m} / \mathrm{s}$. Obviously, with the increase of electrolyte inlet velocity, the rotating driving torque of electrolyte on the impeller increases, and the flow field velocity after impeller rotation also increases. 


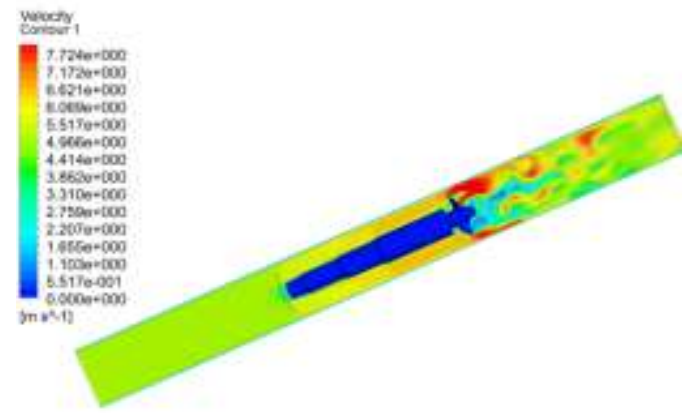

(a) $5 \mathrm{~m} / \mathrm{s}$

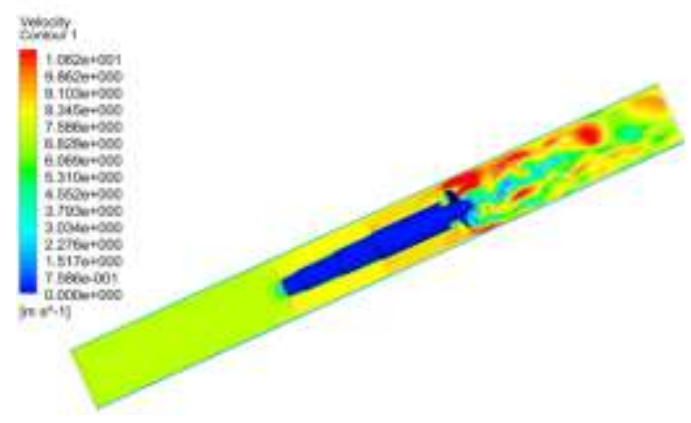

(c) $7 \mathrm{~m} / \mathrm{s}$

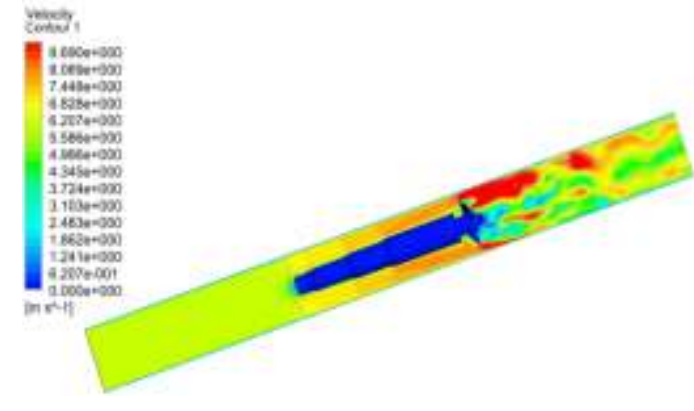

(b) $6 \mathrm{~m} / \mathrm{s}$

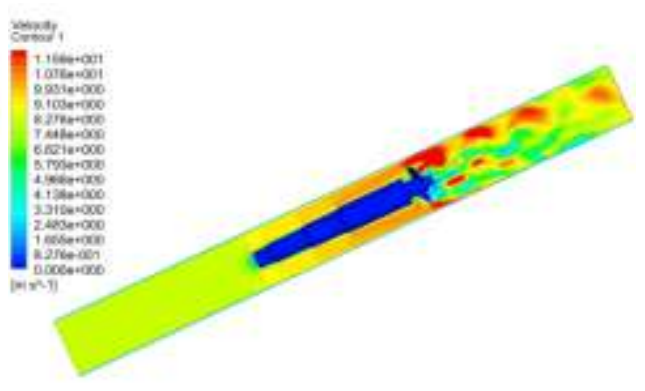

(d) $8 \mathrm{~m} / \mathrm{s}$

Fig. 6 cloud chart of different speeds

In the electrochemical machining process, the tool cathode moves to the prefabricated hole of the workpiece, and the electrolyte entering the cathode body from the hollow pull rod washes the impeller embedded at the tail of the mandrel in the cathode cavity to drive the impeller to rotate. The impeller starts to accelerate under the impact of electrolyte. When the rotating dynamic moment of the impeller reaches equilibrium with the friction resistance moment generated during its movement, it starts to rotate stably. When the minimum electrolyte flow rate is $5 \mathrm{~m} / \mathrm{s}$, define the mass, moment of inertia and other parameters of the impeller by compiling a macro program, import the Fluent calculation solver to calculate the stress change of the impeller, and draw the speed curve according to the output instantaneous speed of the impeller, as shown in Figure 7. From the figure, when the impeller reaches the maximum speed of $4 \mathrm{r} / \mathrm{s}$, it tends to a stable rotation state, At this time, the cathode inner cavity mandrel can be driven to rotate, and the permanent magnet block is embedded on the cathode mandrel, which can realize a stable rotating magnetic field during rotation.

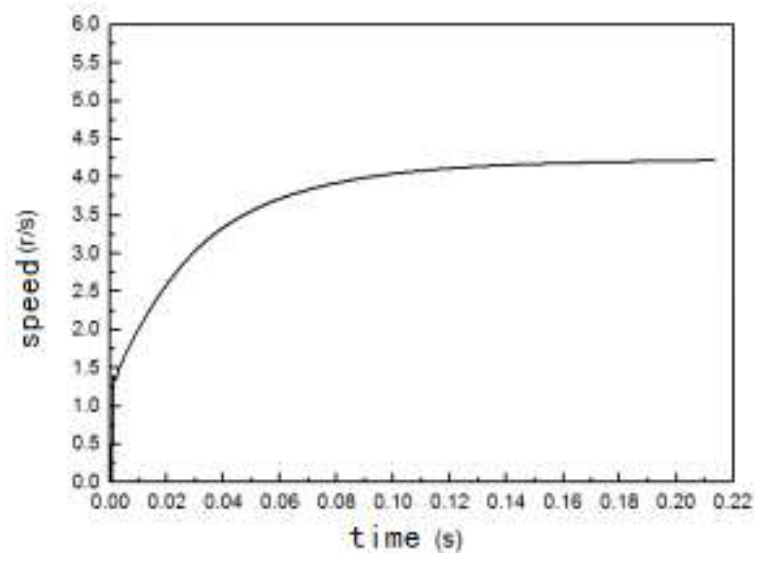


Fig. 7 impeller speed curve

\section{Design of hydrodynamic self driving rotating magnetic field cathode}

The research object of this paper is multi-stage inner conical hole parts, and the section structure is shown in Figure 8. The multi-stage inner taper hole is composed of a straight hole and taper holes with different taper at both ends. The difficulty of this kind of parts is to ensure the taper of the conical surfaces at both ends and the quality of the inner surface of the hole.

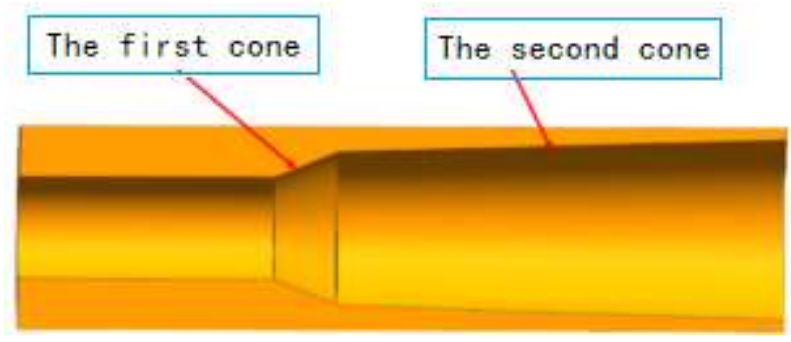

Fig. 8 structure diagram of multi-stage inner conical hole

The cathode of ECM is equivalent to the tool in traditional machining, and its design is the most key link in ECM. In this paper, the moving cathode is used for electrochemical machining of multi-stage inner conical holes. In the cathode, the electrolyte is introduced from the liquid inlet hole at the front end of the cathode body, flows through the cavity between the cathode body and the mandrel, washes the impeller, ejects from the liquid outlet hole on the rear guide and enters the machining gap. The impeller embedded in the tail of the mandrel rotates under the hydraulic self drive of the electrolyte to realize the stable rotation of the mandrel and improve the surface quality of the workpiece. In the machining gap, the electrolyte flows from the large end to the small end of the conical hole of the workpiece to be machined, and the gap flow field is convergent, which solves the problem of non convergence and divergence of the flow field. The structure of hydraulic self driving rotating cathode is shown in Figure 9.

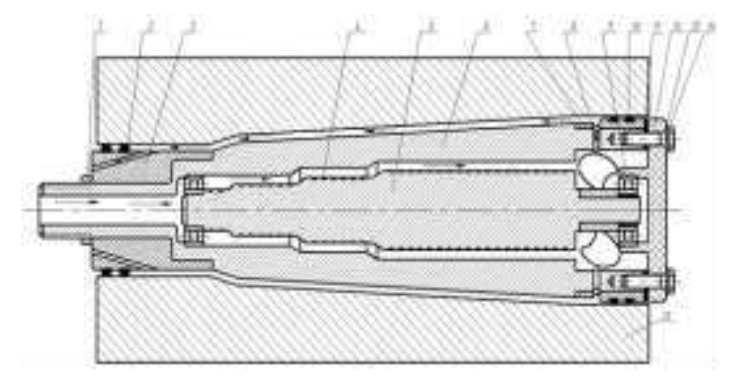

1.Nut, 2.seal ring, 3.Front guidance, 4.Magnetic block, 5.Axis, 6.Cathode body, 7.Post guidance, 8.Impeller, 9.Bearing, 10.Seal ring, 11.Shim, 12.End cap, 13.Washer, 14.Hexagon headed bolt, 15. Workpiece

a)Two dimensional diagram of hydraulic self driving rotating cathode design 


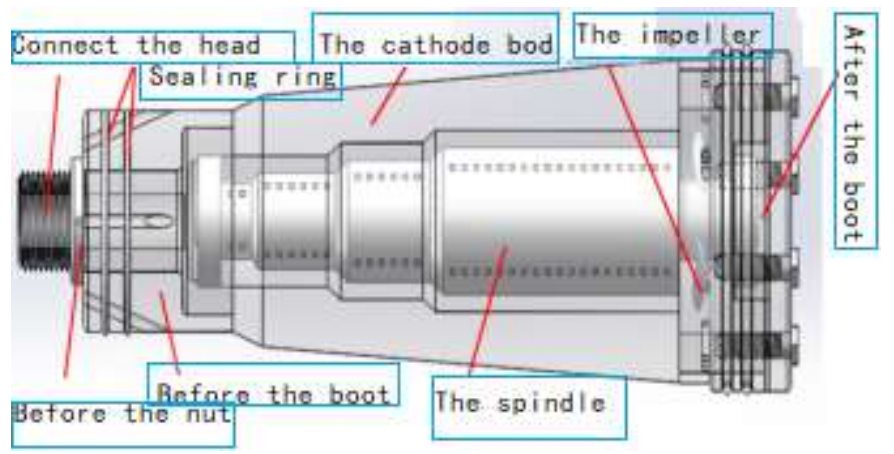

b) Three dimensional model of hydraulic self driving rotating cathode

Fig. 9 Structure diagram of hydraulic self driving rotating cathode

\section{Comparative test of working pairs with and without rotating magnetic field}

The processing technology comparison test equipment for multi-stage inner conical hole parts with and without rotating magnetic field is the horizontal NC ECM system independently developed by our research group, as shown in Figure 10. The system mainly includes: machine bed, power supply system, control system, cooling system, electrolyte circulation system, filtration system, etc.

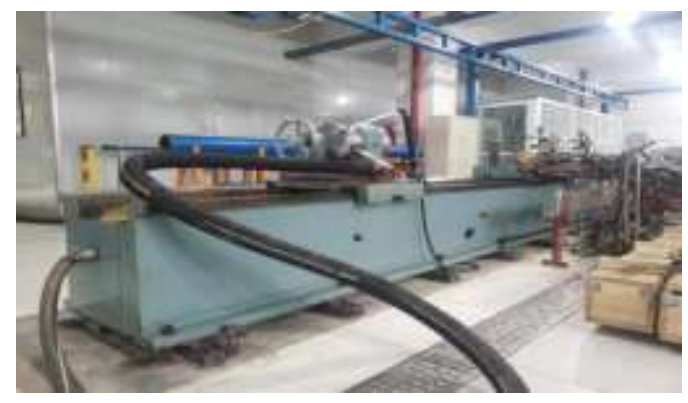

Figure 10 Horizontal NC ECM system independently developed

Under the conditions of processing voltage of $10 \mathrm{~V}$, electrolyte temperature of $30^{\circ} \mathrm{C}$, electrolyte pressure of $1.6 \mathrm{MPa}$ and cathode feed rate of $5 \mathrm{~mm} / \mathrm{min}$, the electrolyte adopts $5 \% \mathrm{NaCl}+$ $15 \% \mathrm{NaNO}_{3}+5 \% \mathrm{NaClO}_{3}$ composite solution, and the cross-section of multi-stage inner conical hole sample processed by cathode without magnetic field on horizontal NC ECM machine tool is shown in Figure 11 .

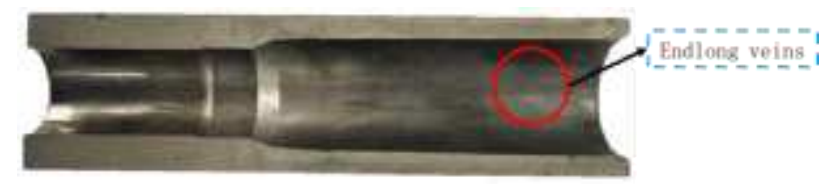

Figure 11 Sample of cathode processing without magnetic field

Because the machining gap is easy to mutate without magnetic field, resulting in uneven gap flow field, there are flow lines on the workpiece surface after machining, and the surface quality is poor.

Under the same processing parameters, the profile of multi-stage inner conical hole sample processed by hydraulic self driving rotating magnetic field composite cathode is shown in FIG. 12 


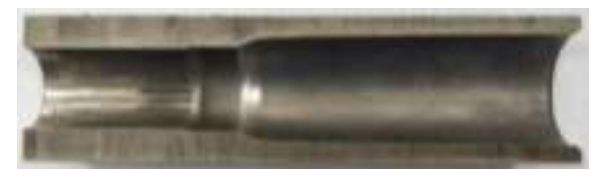

Figure 12 Sample of hydraulic self driving rotating magnetic field composite cathode processing

It can be seen that the multi-stage inner conical hole sample processed by the hydraulic self driving rotating magnetic field composite cathode has no streaking phenomenon. This is due to the influence of the magnetic field, the electromagnetic force in the machining gap changes periodically in the process of magnet rotation, which improves the uniformity of the whole flow field and is conducive to eliminating the streaking phenomenon.

On the on-line cutting machine tool, cut a small piece of the multi-stage inner conical hole sample processed without and with magnetic field, and place it on the white light interferometer ZYGO Plus test platform, as shown in Figure 13, to measure the surface roughness of the multi-stage inner conical hole. The test results are shown in FIG. 14. There are micro flow lines on the surface of the sample processed without magnetic field, and the surface roughness value is $\mathrm{Ra} 0.847 \mu \mathrm{m}$. The surface roughness of the sample under the action of rotating magnetic field is $\mathrm{Ra} 0.437 \mu \mathrm{m}$.

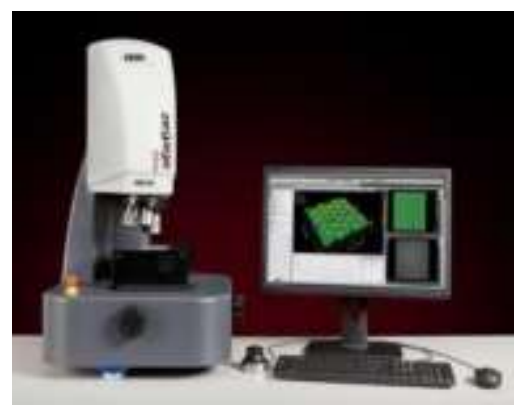

Figure 13 ZYGO PlusWhite light interferometer
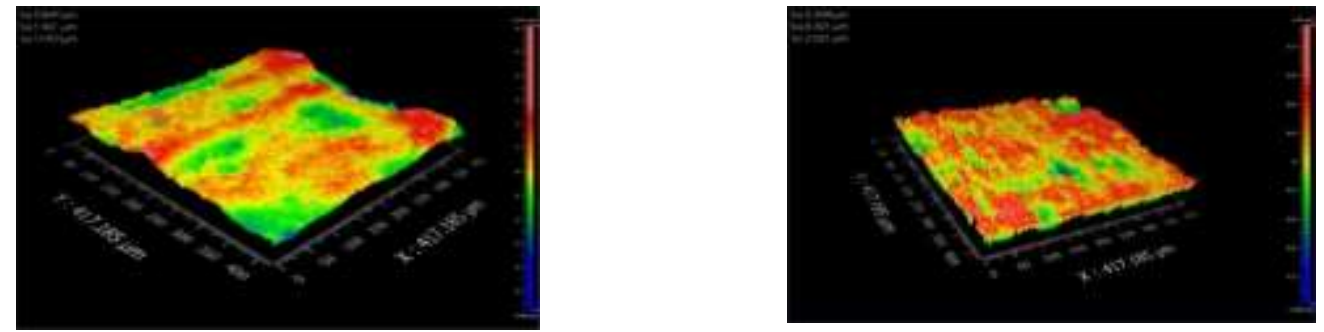

a)Samples processed without magnetic field b)Sample machined by rotating magnetic field

Fig. 14 Surface roughness test results

\section{5 conclusions}

In this paper, for multi-stage inner conical hole ECM, the simulation research of hydraulic self driving rotating flow field is carried out, the hydraulic self driving rotating magnetic field cathode is designed, and the comparative experimental research on multi-stage inner conical hole ECM process with and without rotating magnetic field is carried out. The following conclusions are obtained:

(1) When the number of blades of cathode impeller is 3, the thickness of blades is $0.8 \mathrm{~mm}$ and the minimum electrolyte flow rate is $5 \mathrm{~m} / \mathrm{s}$, the impeller at the tail of cathode mandrel can rotate stably under the impact of electrolyte and improve the uniformity of flow field in machining gap. When the number of blades of cathode impeller is 3 , the thickness of blades is $0.8 \mathrm{~mm}$ and the minimum 
electrolyte flow rate is $5 \mathrm{~m} / \mathrm{s}$, the impeller at the tail of cathode mandrel can rotate stably under the impact of electrolyte and improve the uniformity of flow field in machining gap.

(2) When the processing voltage is $10 \mathrm{~V}$, the electrolyte temperature is $30^{\circ} \mathrm{C}$, the electrolyte pressure is $1.6 \mathrm{MPa}$, the cathode feed rate is $5 \mathrm{~mm} / \mathrm{min}$, and the electrolyte is $5 \% \mathrm{NaCl}+16 \% \mathrm{NaNO}_{3}+4 \% \mathrm{NaClO}_{3}$ composite electrolyte, there is no flow pattern on the inner surface of the processed workpiece, and the surface roughness is $\mathrm{Ra} 0.437 \mu \mathrm{m}$. Compared with ECM without magnetic field, the surface quality of the workpiece is improved by $48.41 \%$.

\section{Authors' contribution}

Lin Tang was the main contributor and corresponding author of the manuscript. Professor Lin Tang designed the research process, and led Xingchen Ge and Kaige Zhai to establish a hydraulic self driving rotating flow field model and simulate the flow field, optimizing the structure of cathode tail blade. Lifeng Zhang and Chengjin Shi carried out a comparative experimental study on the multi-stage inner conical hole ECM process with and without rotating magnetic field.

\section{Funding information}

This work was financially supported by Shaanxi Province Key Research and Development projects ( Grant No. 2020GY-153), Scientific research program for Youth Innovation Team Construction of Shaa nxi Provincial Department of Education (No: 21JP054), and Shaanxi University Youth Innovation Tea m Project (Grant No: 20201020).

\section{Availability of data and materials}

All data generated or analysed during this study are included in this published article.

\section{Declarations}

\section{Ethics approval}

Not applicable.

\section{Consent to participate}

Not applicable.

\section{Consent for publication}

Not applicable.

\section{Conflict of interest}

The authors declare no competing interests.

\section{References}

[1] Hassan El-Hofy (2019) Vibration-assisted electrochemical machining: a review. Int J Adv Manuf Technol 105(1-4):579-593.

[2] Schubert N, Schneider M, Michaelis A, et al (2018) Electrochemical machining of tungsten carbide 22(3):859-868.

[3] Zhang CY, Zhang YJ, Chen XL, et al (2018) Investigation of the electrochemical dissolution behavior of 
tungsten during electrochemical machining. Int J Adv Manuf Technol 97:9-12.

[4] Wang YD, Xu ZY, Zhang A (2020) Anodic characteristics and electrochemical machining of two typical $\gamma$-TiAl alloys and its quantitative dissolution model in NaNO3 solution. Electrochimica Acta.

[5] Tang L, Yang S (2013) Experimental investigation on the electrochemical machining of 00Cr12Ni9Mo4Cu2 material and multi-objective parameters optimization. Int J Adv Manuf Technol 67(9): 2909-2916.

[6] Qu NS, Fang XL, Zhu D.(2013) Enhancement of surface roughness in electrochemical machining of Ti6Al4V by pulsating electrolyte. Int J Adv Manuf Technol, 69(9-12): 2703-2709.

[7] Burger M, Koll L, Werner EA, et al. (2012) Electrochemical Machining Characteristics and Resulting Surface Quality of the Nickel-base Single-crystalline Material LEK94. Journal of Manufacturing Processes, 14:62-70.

[8] Selvarajan L.(2020) Investigations on electrochemical machining (ECM) of Al7075 material using copper electrode for improving geometrical tolerance. Materials Today: Proceedings.

[9] Fan ZJ,Ma BJ,Wang TC. (2006) Embedded magnetic circuit of the electrochemical machining technology. Journal of mechanical engineering, (02):96-100. （in Chinese )

[10] Lin Mingyuan, Tsai TH, Hourng LW, Wang Weikai. (2019) The effects of magnetic field and ethanol addition on the electrochemical discharge machining. Int J Adv Manuf Technol, 105(5-6):2461-2467.

[11] Ayyappan, S, Sivakumar K, Kalaimathi M. (2015) Electrochemical machining of 20MnCr5 alloy steel with magnetic flux assisted vibrating tool. Proceedings of the Institution of Mechanical Engineers, Part C: Journal of Mechanical Engineering Science, 231(10):1956-1965.

[12] Pa PS.(2009) Super finishing with ultrasonic and magnetic assistance in electrochemical micro-machining. Electrochimica Acta, (54):6022-6027.

[13] Fan ZJ, Wang GG, Tang L, Wang TC .(2010) Design of device and experiment on magnetic field assisted electrochemical machining. Journal of Mechanical Engineering 46(1):194-198. （in Chinese）.

[14] Ning SK,Chen JY.Ma BJ.(2016) Magnetic composite electrochemical machining flow field distribution features of numerical simulation. Mechanical design and manufacturing engineering, 45(04):85-87. (in Chinese)

[15] Tang L, Gan WM.(2014) Experiment and simulation study on concentrated magnetic field-assisted ECM S-03 special stainless steel complex cavity. Int J Adv Manuf Technol, 72:685-692.

[16] Tang L,Guo YF.(2013) Assembled magnetic complex cavity research of electrolytic machining accuracy. Machining and mold, (02):50-54. (in Chinese)

[17] Li L, Ma BJ, Cheng PY, et al.(2018) A comparative study on the effects of magnetic field on the anode surface roughness in the forward and reverse electrolyte feed pattern during electrochemical machining. Int J Adv Manuf Technol, 101(5-8):1635-1650.

[18] Li L, Ma BJ.(2018) Effect of magnetic field on anodic dissolution in electrochemical machining. Int J Adv 
Manuf Technol, 94:1177-1187.

[19] Li L, Ma BJ, Cheng PY, et al. (2019) Effect of magnetic field on the electrochemical machining localization. Int J Adv Manuf Technol, (102):949-956.

[20] Qiu Y,Zhang WM,Zhang ZJ.(2013) A rotating magnetic field -high frequency group pulse electrochemical composite processing experimental study. Journal of Beijing institute of technology, 4(33):353-356. （in Chinese )

[21] Liu GX, Zhang YJ, Natsu W.(2019) Influence of electrolyte flow mode on characteristics of electrochemical machining with electrolyte suction tool. International Journal of Machine Tools and Manufacture, 142:66-75.

[22] Liu GQ,Liu L,He CK.(2018) Electrochemical machining process more physical field coupling simulation and experiment research. Mechanical design,35(12):29-35. （in Chinese）

[23] Hu XY, Zhu D, Li JB, et al.(2019) Flow field research on electrochemical machining with gas film insulation. Journal of Materials Processing Tech.(267):247-256.

[24] Li JZ, Wang DY, Zhu D, et al. (2020)Analysis of the flow field in counter-rotating electrochemical machining. Journal of Materials Processing Tech,275.

[25] Chen YL, Zhou XC, Chen PX.(2019) Electrochemical machining gap prediction with multi-physics coupling model based on two-phase turbulence flow. Chinese Journal of Aeronautics.

[26] Chen YL.(2015) Variable cross-section bending of electrochemical machining tooling fluid-structure coupling analysis and optimization design. The Chinese mechanical engineering society of special processing branch.The 16th national conference on special processing academic (on). The Chinese mechanical engineering society (CMES) special processing branch: the Chinese mechanical engineering society, 5. (in Chinese)

[27] ChenYL,Zhou XC,Chen PH.(2019) Based on the SST turbulence model electrochemical machining temperature field numerical simulation research. Journal of mechanical engineering, 55(17):215-221. （in Chinese )

[28] Zhou XC,Cao CY,Lin H.(2018) Electrochemical machining process parameter optimization of the simulation. Journal of qiqihar university (natural science edition), 34(03):43-45. （in Chinese )

[29] Ji L,Zhang Y,Guo ZC.(2019) Spiral tube electrode edm small hole - electrolytic combination processing numerical simulation and experimental analysis. Journal of nanjing university of technology (natural science edition), 41(06):679-687. (in Chinese )

[30] He YF,Lu WZ,Gan WM.(2016) Titanium alloy square hole electrochemical machining physical field coupling research more. Journal of south China university of technology (natural science edition), 44(05):110-116. (in Chinese)

[31] He YF,Lu WZ,Gan WM.(2016) Based on the analysis of the electric field electrochemical machining titanium alloy round hole forming research. The Chinese mechanical engineering, 27(11):1461-1466. （in 


\section{Chinese )}

[32] Wang F,Zhao JS,Liu DM.(2019) Titanium alloy deep narrow slot controllable vibration assisted electrochemical machining experiments. The Chinese mechanical engineering,30(20):2395-2402. (in Chinese )

[33] Li PT,Jing Q,Zhang YB.(2020) Wire electrode superposed jet electrochemical machining simulation and experimental research. Journal of Harbin Institute of Technology university, 52(01):178-185. （in Chinese ) 